\title{
Die augmentierte iliosakrale Schraube bei Beckenfrakturen im Alter
}

Niklas Grüneweller, Thomas Fuchs, Dirk Wähnert, Michael J. Raschke

\section{Epidemiologie}

Im Jahr 2010 traten 68\% der klinisch diagnostizierten Beckenringfrakturen in der Gruppe der über 65-Jährigen auf. Dabei lag der Hauptanteil in der Altersgruppe der 85- bis 90-Jährigen [2,5]. Die Patientengruppe der über 60-Jährigen stellt insgesamt ein sehr heterogenes Kollektiv dar, deshalb müssen Komorbiditäten, Selbstständigkeit, Patientenansprüche und -aktivität in die Therapieentscheidung eingeschlossen werden. Auch die Prävalenz der Osteoporose spielt bei der alters- und geschlechtsspezifischen Verteilung der Beckenringfrakturen eine entscheidende Rolle. In der Altersgruppe der 65- bis 74Jährigen konnte eine Osteoporoseprävalenz von $32 \%$ bei Frauen und von $8 \%$ bei Männern festgestellt werden. Mit weiter zunehmendem Alter erhöht sich diese Prävalenz auf $48 \%$ (bei Frauen) bzw. 18\% (bei Männern). Jede 4. Frau und jeder 17. Mann $>50$ Jahre ist an Osteoporose erkrankt, mehr als die Hälfte der Betroffenen erleidet innerhalb von 4 Jahren mindestens eine osteoporosebedingte Fraktur [4]. Diese Daten unterstreichen die weibliche Prädominanz auch bei Beckenfrakturen im Alter > 65 Jahre mit 57\% Anteil weiblicher Patienten [2,5]. Insgesamt ist die osteoporotische Beckenringfraktur eine unterschätze, allerdings komplikationsbehaftete osteoporotische Fraktur.

\section{Definition}

Die Beckenringfraktur des älteren Patienten kann mit verschiedenen Begrifflichkeiten, die sich in ihrer Definition gering unterscheiden, beschrieben werden.

Die „Insuffizienzfraktur des Beckenrings“ ist relativ eng definiert: Es handelt sich dabei um eine Fraktur verursacht durch das Einwirken einer physiologischen Kraft auf (osteoporotisch) veränderten Knochen.

Im Gegensatz dazu erlaubt der Begriff der „osteoporotischen Beckenringfraktur" definitionsgemäß auch das Einwirken nicht physiologischer Kräfte (Traumamechanismus). Allerdings muss man festhalten, dass diese Kräfte nicht mit denen vergleichbar sind, die beim jungen Patienten typischerweise zu Beckenringfrakturen führen (Hochrasanztrauma).
Ein weiterer, in jüngster Zeit zunehmend gebräuchlicher Begriff für diese Verletzungsentität ist die „fragility fracture of the pelvis“ („fragility“ = Englisch für „Brüchigkeit“ bzw. „Gebrechlichkeit“; Abkürzung: FFP, siehe Abschnitt „Klassifikation“). Dieser Begriff fasst die zugrunde liegenden prädisponierenden knöchernen Veränderungen zusammen und beinhaltet als Frakturauslöser sowohl die physiologische als auch nicht physiologische Krafteinwirkung auf Knochen reduzierter Qualität.

Wir bevorzugen die Bezeichnung der FFP, da die Fraktureinteilung nach physiologischer bzw. nicht physiologischer Kraft anamnestisch häufig nicht zu differenzieren ist und für die weitere Diagnostik und Therapie von untergeordneter Relevanz ist.

Das Patientenalter wird von keinem der o.g. Begriffe berücksichtigt.

\section{Ätiologie}

Die Beckenfraktur des älteren Patienten unterscheidet sich wesentlich von der des jüngeren. Beim jungen Patienten sind Beckenfrakturen Folge eines Hochrasanztraumas (z. B. Verkehrsunfall, Sturz aus großer Höhe, Polytrauma) und meist vergesellschaftet mit intra- und extrapelvinen Begleitverletzungen sowie einer erheblichen Frakturdislokation. Diese Umstände erklären auch die erhöhte Morbidität und Mortalität dieser Verletzungen beim jungen Patienten sowie die Indikation zur akuten operativen Versorgung und teilbelastender bzw. entlastender Nachbehandlung im Anschluss.

Die Beckenringfraktur des älteren Patienten entsteht überwiegend entweder ohne erinnerliches Trauma oder bei Niedrigenergieunfällen (Sturz zu ebener Erde mit vertikaler Krafteinwirkung auf das Gesäß oder lateraler Krafteinwirkung auf die Hüfte). Durch diesen Unfallmechanismus fehlen signifikante Weichteilverletzungen, und die Frakturen sind häufig nur gering disloziert (Cave: Diagnostik). In Ausnahmefällen kann auch beim älteren Patienten ein Hochrasanztrauma Ursache für eine Beckenverletzung sein. Aufgrund der geringeren funktionellen Reserven besteht dann ein deutlich höheres Mortalitätsrisiko [1]. 


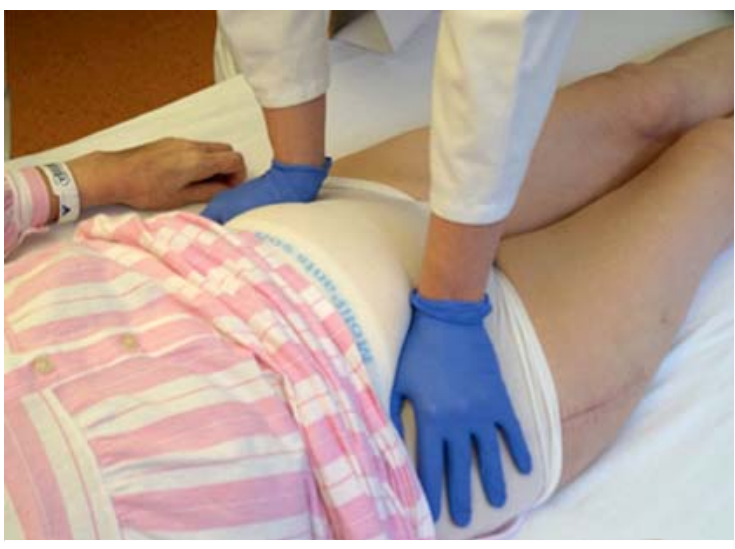

- Abb. 1 Untersuchung der Beckenstabilität mit Schmerzprovokation im Bereich des vorderen und hinteren Beckenrings durch Aufklappen.

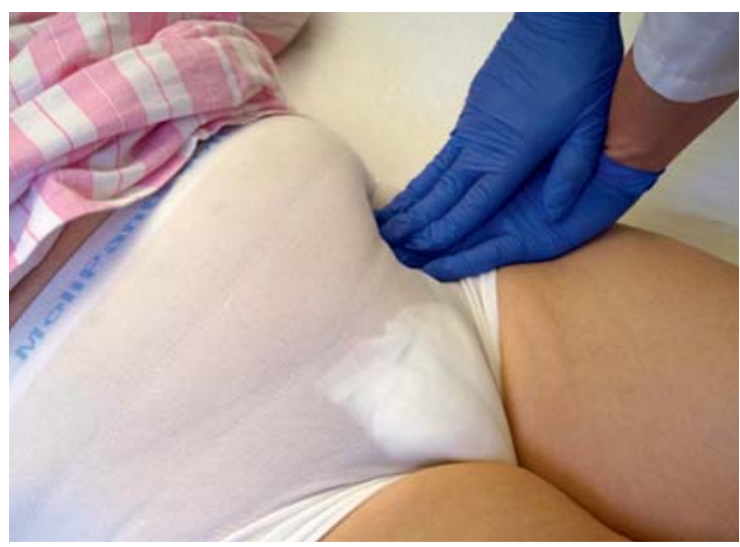

Abb. 3 Untersuchung des oberen Schambeinasts mit vorsichtiger Druckschmerzprovokation.

Wesentlichen Einfluss auf das Entstehen einer Beckenringfraktur beim älteren Patienten haben die Sturzneigung und die Knochenqualität. Mit zunehmendem Alter steigt die Sturzneigung, so liegt diese bei den > 65-Jährigen bei ca. 30\% und steigt im weiteren Altersverlauf an [10]. Das Sturzrisiko ist durch Komorbiditäten wie HerzKreislauf-Erkrankungen (Hypertonie, Hypotonie, Herzrhythmusstörungen), Sehstörungen, neurologische Erkrankungen (Morbus Parkinson, Demenz, Polyneuropathie bei Diabetes mellitus, hospitalisationsbedingtes Delir), Abnahme der muskulären Kraft und der Koordination sowie durch Medikamenteneinnahme (meist Polymedikation mit Wechselwirkungspotenzial) bedingt und benötigt ein interdisziplinäres Patienten-Assessment sowie ein entsprechend angepasstes multimodales Therapiemanagement $[2,12]$.

Die strukturelle Schwächung des Knochens kann multifaktoriell bedingt sein. Hauptursache ist allerdings die primäre Osteoporose (hauptsächlich postmenopausale

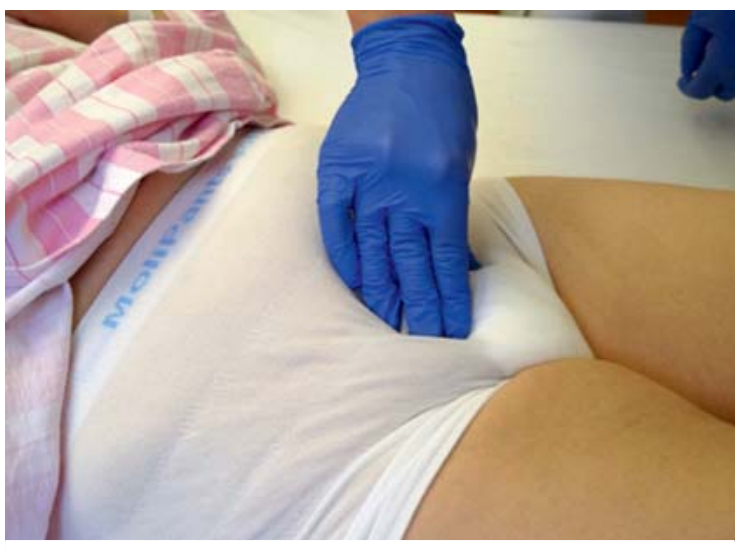

- Abb. 2 Untersuchung der Symphyse mit vorsichtiger Druckschmerzprovokation.

Frauen). Des Weiteren sind aber auch Ursachen der sekundären Osteoporose abzuklären bzw. zu behandeln (z.B. Medikation mit Kortikosteroiden, Vitamin-D-Mangel, Immobilisation, Bettlägerigkeit) [2,6]. Auch Begleiterkrankungen bzw. -behandlungen können mit einer Schwächung des Knochens einhergehen und müssen berücksichtigt werden. Hier sind die rheumatoide Arthritis, der Zustand nach Bestahlung der Beckenregion, Tumoren/Metastasen und Knochenentnahme im Bereich des hinteren Beckenrings zu nennen [2,6].

\section{Anamnese und Untersuchung}

Typischerweise liegt bei älteren Patienten mit einer Beckenringfraktur eine positive Sturzanamnese vor, oftmals wird ein Sturz bagatellisiert oder aufgrund von Nebenerkrankungen, wie Demenz, nicht erinnert. Gegebenenfalls ist diesbezüglich eine gezielte Fremdanamnese der Angehörigen oder der Pflegenden zielführend. Die klinische Symptomatik variiert je nach Frakturlokalisation, so sind Schmerzen im Bereich der Leisten oder im Schritt charakteristisch bei Schambeinastfrakturen, dagegen ist ein „tief lumbaler“ Schmerz typisch bei Sakrumfrakturen. Gemein ist diesen Frakturen der immobilisierende Schmerzcharakter.

Die körperliche Untersuchung des älteren Patienten ( Abb. 1-5) kann, je nach Alter und Nebenerkrankungen (insbesondere Demenz), hinsichtlich der Sensitivität des Vorliegens einer Beckenringfraktur eine Herausforderung darstellen. Der oben beschriebene charakteristische Druckschmerz über der Frakturlokalisation ( $\mathbf{A}$ bb. 2, 3 und 5) ist insbesondere im Bereich des hinteren Beckenrings von Differenzialdiagnosen wie Wirbelkörperfrakturen der Lendenwirbelsäule (z.B. osteoporotische Sinterungsfrakturen) oder Bandscheibenvorfällen mit radikulärer Schmerzsymptomatik abzugrenzen. Erschwerend 


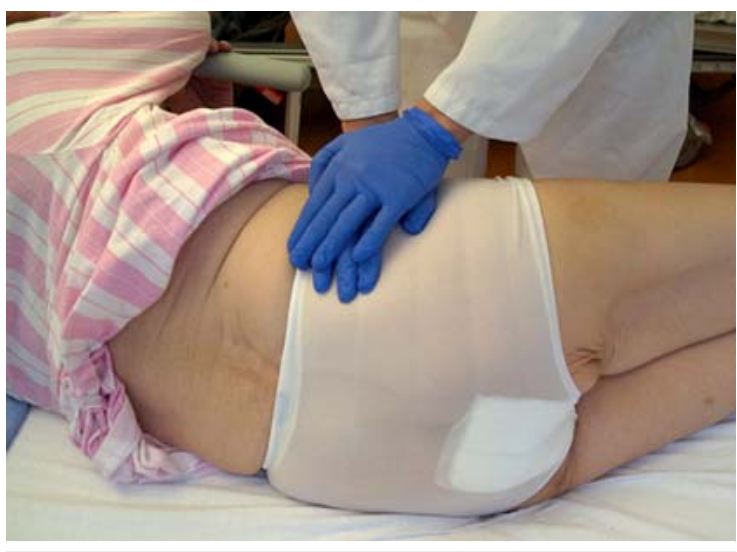

- Abb. 4 Kompression des Beckens in Seitenlage zur Schmerzprovokation insbesondere dorsal.

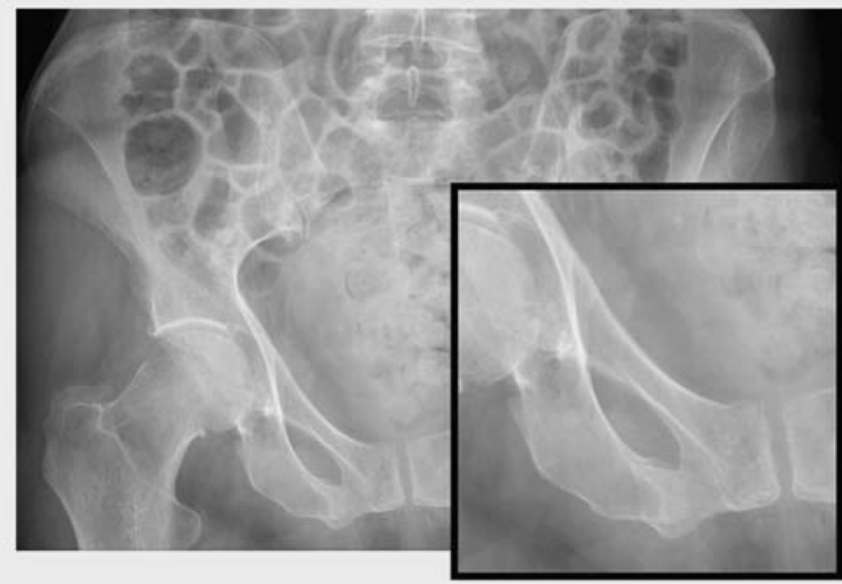

- Abb. 6 Konventionelles Röntgenbild des Beckens im anterior-posterioren Strahlengang einer 91-jährigen Patientin mit immobilisierenden Schmerzen ventral sowie dorsal nach Sturz auf das Gesäß. Fraktur des unteren Schambeinasts, dorsaler Beckenring insuffizient beurteilbar bei Darmgasüberlagerungen.

kann bei Frakturen des hinteren Beckenrings eine pseudoradikuläre Schmerzsymptomatik vorliegen. Diesbezüglich ist die Integrität der Nervenwurzeln L V bis S V aufgrund der potenziellen Gefährdung durch die anatomische Nähe zur Fraktur zu untersuchen und zu dokumentieren.

\section{Diagnostik}

Frakturen des vorderen Beckenrings sind in vielen Fällen durch ein konventionelles Röntgenbild vom Becken im anterior-posterioren Strahlengang zu diagnostizieren

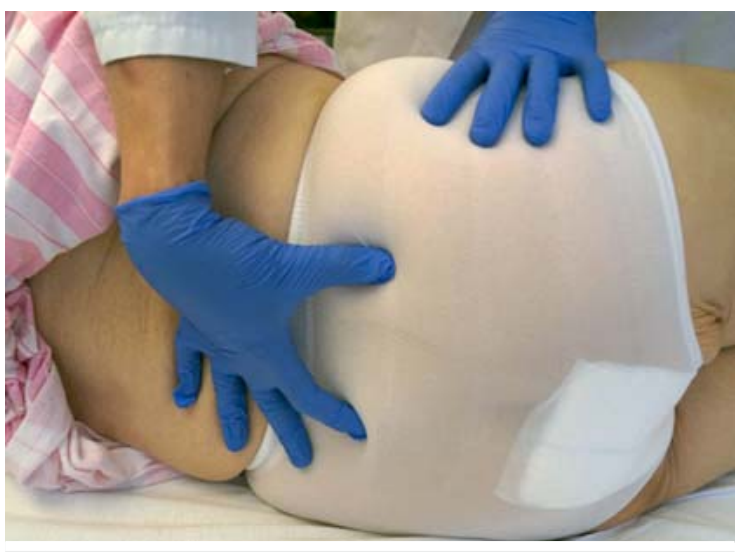

- Abb. 5 Untersuchung des Sakrums mit vorsichtiger Druckschmerzprovokation paramedian über den Alae (insbesondere bei Schmerzen in dieser Untersuchung ist eine Verletzung des hinteren Beckenrings sehr wahrscheinlich).

( $\triangleright$ Abb. 6). Dahingegen ist die Beurteilbarkeit des hinteren Beckenrings, besonders bei gering dislozierten Frakturen und beim älteren Patienten, deutlich eingeschränkt. Besonderen Einfluss haben diesbezüglich die reduzierte Knochendichte, degenerative Veränderungen sowie Überlagerungen von Darminhalt bzw. Darmgasen ( $\bullet$ Abb. 6).

In klinischen Studien hat sich gezeigt, dass bei einer nativradiologisch nachgewiesenen Fraktur des vorderen Beckenrings in 54-97\% (!) der Fälle auch eine knöcherne Läsion im Bereich des hinteren Beckenrings vorliegt [9, 11]. Bei klinischem Verdacht auf eine Fraktur in dieser Region sollte daher eine computertomografische Diagnostik des Beckens forciert werden ( $\mathbf{A b b}$. 7-10).

\section{Merke}

Bei Frakturen des vorderen Beckenrings ist eine Fraktur im Bereich des hinteren Beckenrings auszuschließen.

In der CT-grafischen Diagnostik kann sehr häufig im Bereich der Alae des Os sacrum ein Bereich mit erheblich reduzierter Knochenstruktur nachgewiesen werden ( $\bullet$ Abb. 9 und 10); teilweise ist in diesen sog. „alar voids“ („void“ = Englisch für „Leere“) wenig Knochengewebe nachweisbar. Diese Bereiche stellen eine Prädilektionsstelle für das Auftreten von Frakturen dar ( $\bullet$ Abb. 11).

\section{Frakturmorphologie}

Der Unfallmechanismus und das Patientenalter haben einen entscheidenden Einfluss auf die Frakturmorphologie. Generell nimmt die Frakturschwere bzw. -komplexität im Alter ab. Dies spiegelt sich in der erschwerten nativradiologischen Diagnostizierbarkeit von Frakturen vor 
allem des hinteren Beckenrings aufgrund des geringeren Dislokationsgrads wider. Bei geringer Dislokation und der entsprechend großen knöchernen Kontaktfläche sind diese Frakturen eher als „stabil“ anzusehen. Geringer ausgeprägt sind dementsprechend auch traumaassoziierte Verletzungen der intrapelvinen Gefäße oder Organe bzw. die Beteiligung von nervalen Strukturen. Grundsätzlich können aber alle Frakturmorphologien, auch dislozierte Frakturen, auftreten. Eine isolierte Verletzung des vorderen oder hinteren Beckenrings ist selten. Beim Vorliegen einer altersbedingten Verknöcherung der Iliosakralgelenksfuge treten Frakturen gelegentlich parallel zu dieser auf, wodurch transalare bzw. transforaminale Frakturen im Bereich des Sakrums oder eine transiliakale Fraktur resultieren.

Die typische Fraktur des hinteren Beckenrings beim alten Patienten liegt jedoch im Bereich der Ala des Os sacrum vertikal zwischen Neuroforamenebene und Iliosakralgelenksfuge.

Liegt zwischen Trauma und fokussierter Diagnostik ein längeres Zeitintervall (z. B. durch Bagatellisierung eines Niedrigenergietraumas) oder wurde eine protrahierte oder insuffiziente Therapie durchgeführt, so ist eine Dislokation im zeitlichen Verlauf oder sogar die Ausbildung einer Pseudarthrose möglich. Die frühere Annahme, dass Beckenringfrakturen im Alter aufgrund der geringen Dislokation immer als stabil anzusehen sind und daher alle einer konservativen Therapie zugeführt werden können, ist heutzutage kritisch zu sehen.

\section{Klassifikation}

Die Anwendbarkeit der geläufigen Klassifikationen für Beckenringfrakturen (Tile-, AO/OTA-, Young-Burgess-Klassifikation) ist beim alten Patienten eingeschränkt, da aufgrund der besonderen Umstände (Niedrigenergietrauma, reduzierte Knochenqualität, degenerative Veränderungen) eine gänzlich andere Frakturmorphologie zu finden ist.

Eine von Rommens et al. publizierte CT-basierte Klassifikation unterteilt die Beckenringfrakturen bei Patienten > 65 Jahre in 4 Gruppen aufsteigend nach der Frakturinstabilität. Gleichzeitig gibt diese hierbei Empfehlungen zur Therapie in Abhängigkeit von der Instabilität (konservative vs. minimalinvasive vs. offen operative Therapie). Eine Fraktur kann im zeitlichen Verlauf bei inadäquater Behandlung in die nächsthöhere Instabilitätsgruppe übergehen ( $\triangleright$ Tab. 1 und Abb. 12) [8].

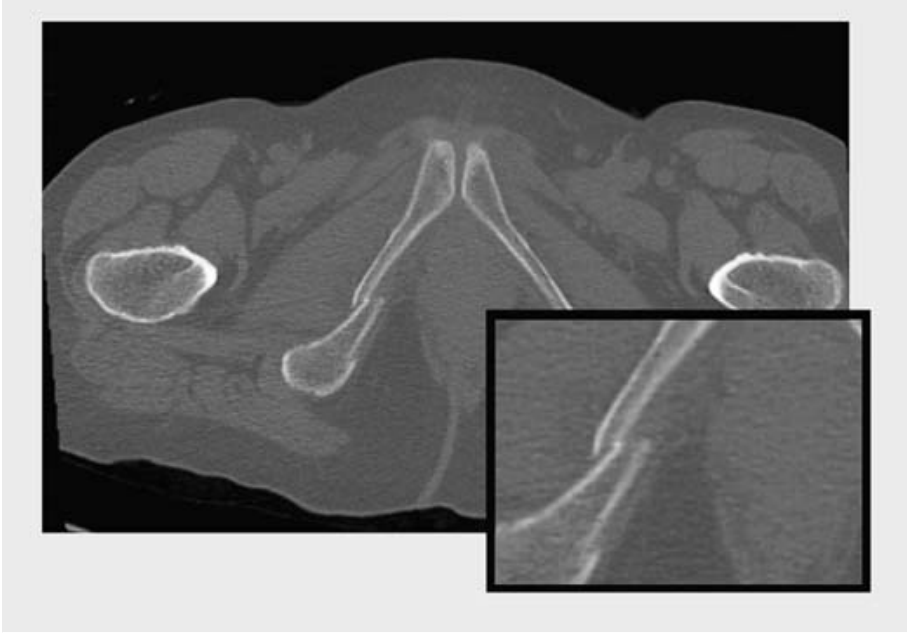

- Abb. 7 Axiale CT-Untersuchung der unteren Schambeinastfraktur rechts aus $>$ Abb. 6.

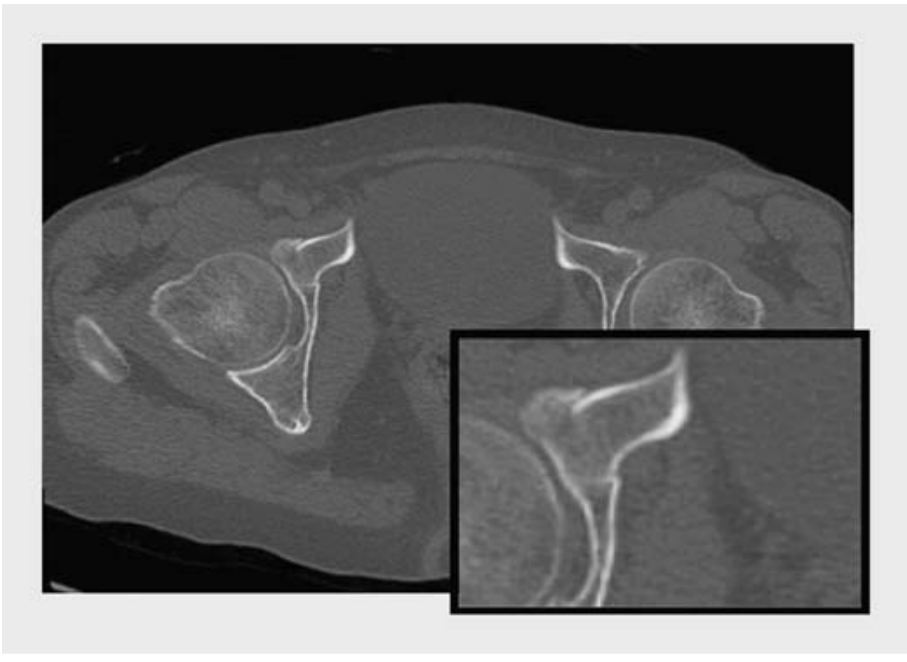

- Abb. 8 Die axiale CT-Untersuchung zeigt zusätzliche azetabulumnahe Fraktur des oberen Schambeinasts.

\section{Therapie}

Merke

Das grundsätzliche Therapieziel bei Beckenringfrakturen im höheren Alter sollte die Wiederherstellung der ursprünglichen Funktionalität und Mobilität sein [7].

Für die Wiederherstellung der ursprünglichen Funktionalität und Mobilität kommen je nach Frakturmorphologie verschiedene Therapieverfahren infrage. Unter Berücksichtigung der individuellen Komorbiditäten und insbesondere der individuellen Vorgeschichte sollte möglichst die schonendste Therapie angestrebt werden, die trotz- 


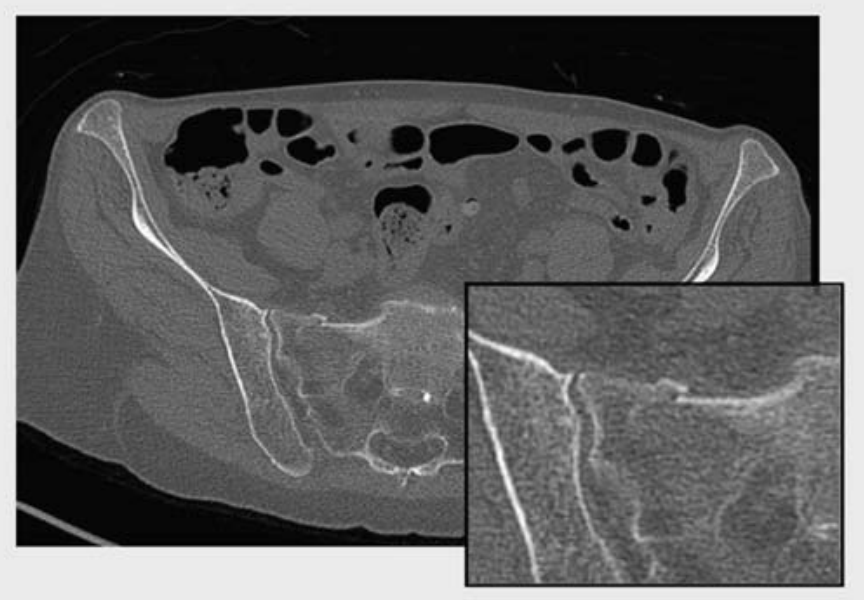

- Abb. 9 Die axiale CT-Untersuchung ermöglicht die suffiziente Beurteilung des hinteren Beckenrings und zeigt eine zusätzliche Fraktur des Sakrums zwischen Neuroforamenebene und Iliosakralgelenksfuge.

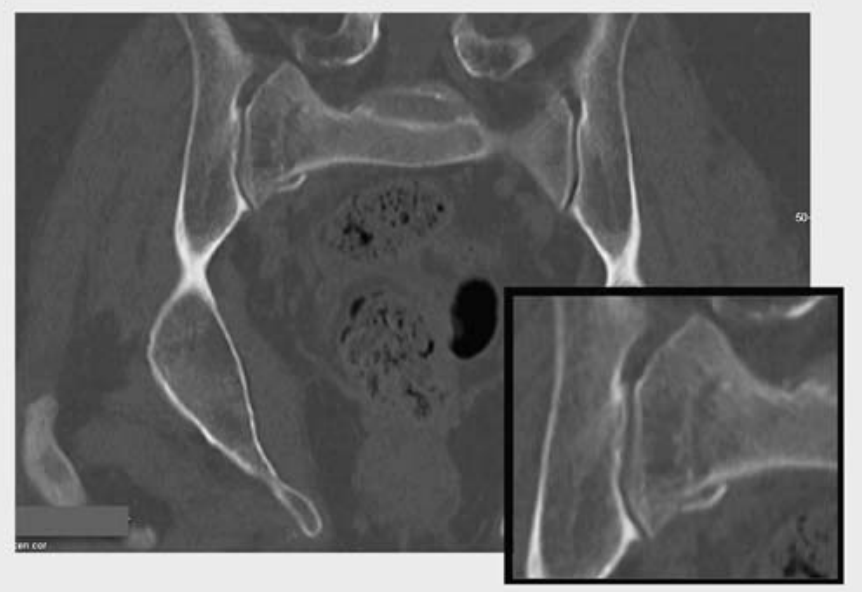

- Abb.10 Entsprechende koronare CT-Untersuchung zeigt eine Fraktur des hinteren Beckenrings parallel zur Iliosakralgelenksfuge.

dem noch eine frühzeitige Mobilisation des Patienten erlaubt.

Die Primärtherapie von Beckenringfrakturen älterer Patienten umfasst eine altersgerechte und angepasste suffiziente Schmerztherapie in Kombination mit physiotherapeutischer Mobilisation. Parallel dazu sollte eine osteoporotische Basisdiagnostik und leitliniengerechte Osteoporosetherapie eingeleitet werden (suffiziente Kalzium-/ Vitamin-D $-D_{3}$-Substitution und ggf. Bisphosphonattherapie; siehe DVO-Leitlinie 2014 unter http://www.dv-osteologie.org/dvo_leitlinien/osteoporose-leitlinie-2014).
Bei frustraner konservativer Therapie kommen verschiedene operative Eingriffe zur Anwendung, dabei sollte minimalinvasiven Verfahren der Vorzug gegeben werden.

Für die minimalinvasive Stabilisierung des hinteren Beckenrings bietet sich die Technik der perkutanen iliosakralen Schraubenosteosynthese an. Bei reduzierter Knochenqualität kann die Verankerung der Schraube im Sakrum durch eine Zementaugmentation der Schraubenspitze verbessert werden. Bei dieser Schraubenaugmentation kommen kanülierte und spitzenperforierte Implantate zur Anwendung. In der eigenen Praxis haben sich Schrauben mit fixierter mobiler Unterlegscheibe und speziellem Zementapplikationssystem bewährt (Fa. Marquardt Medizintechnik, Spaichingen, Deutschland, • Abb. 13 und 14); bei einer ggf. erforderlichen Implantatentfernung muss hier die Unterlegscheibe nicht einzeln geborgen werden.

Knöcherne Verletzungen im Bereich des vorderen Beckenrings können durch einen minimalinvasiv eingebrachten supraazetabulären Beckenfixateur adressiert werden. Die klassischen Indikationen für eine solche Therapie sind FFP-2-Verletzungen ( $\bullet$ Abb. 12 und Tab. 1), also akut aufgetretene nicht bzw. gering dislozierte Frakturen des hinteren Beckenrings (Sakrum) mit oder ohne knöcherne Beteiligung des vorderen Beckenrings nach frustraner konservativer Therapie. Zusätzlich können FFP-3-Verletzungen ohne grobe Dislokation und ohne transiliakale Frakturmorphologie in dieser Art und Weise versorgt werden. Weiterhin ist die Verwendung von zementaugmentierten iliosakralen Schrauben auch als Teil einer spinopelvinen Osteosynthese sinnvoll, um maximale Konstruktstabilität zu erreichen. Dies ist bei hochgradigen dorsalen Instabilitäten (FFP-4-Verletzungen) erforderlich.

Bei komplexen Instabilitäten oder protrahiertem Verlauf kommen als Stabilisierungsverfahren zusätzlich die Plattenosteosynthesen im Bereich des vorderen (Symphyse, Schambeinäste) oder hinteren Beckenrings (überbrückende winkelstabile ilioiliakale Platte, transiliakaler Fixateur interne) zur Anwendung. Diesen Osteosyntheseverfahren ist gemein, dass sie durch einen offenen chirurgischen Zugang eingebracht werden oder prominent unter den Weichteilen zu liegen kommen, wodurch sich das Komplikationsrisiko für z. B. Wundheilungsstörungen und Infektionen erhöht.

\section{Operative Technik}

Die üblichen präoperativen Vorbereitungen müssen bei diesem Eingriff abführende Maßnahmen zur Verbesserung der intraoperativen Röntgendarstellung beinhalten. Zur Operation wird der Patient auf dem Rücken auf einem Carbontisch gelagert. Eine 4-Punkt-Abdeckung ist meist ausreichend, da nur selten Repositionsmanöver 


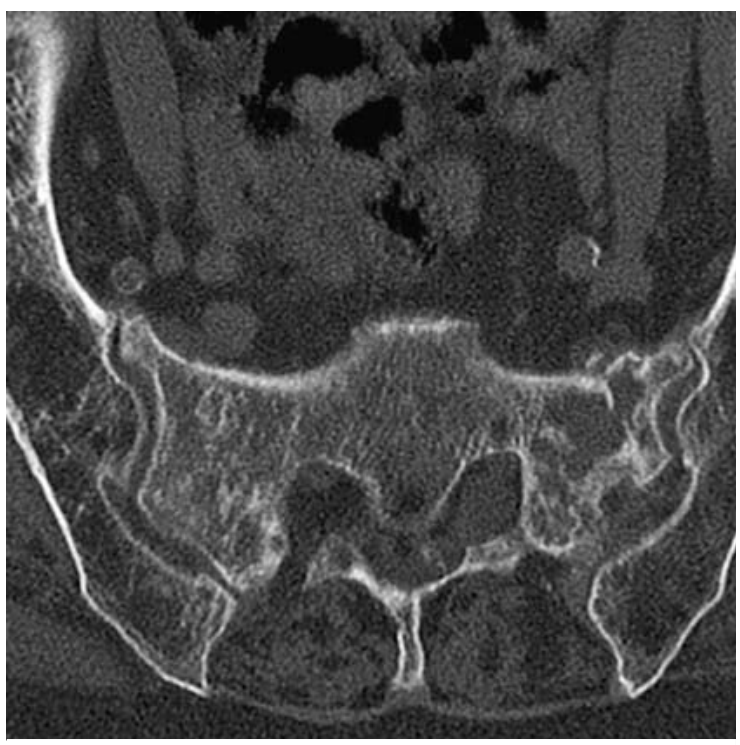

- Abb. 11 Axiale Schnittbildgebung eines exemplarischen Sakrums mit deutlich sichtbarem „alar void“ linksseitig und hier hindurch verlaufender Fraktur.

durchgeführt werden müssen. Zu beachten ist, dass auch die Unterseite des Operationstischs sowie die Säule steril abgedeckt werden, um ein freies steriles Bewegen des Röntgenbildwandlers lateral, a.-p. sowie in Inlet- und Outlet-Projektion zu ermöglichen.
In der von Gänsslen et al. beschriebenen perkutanen Technik [3] wird nun der Führungsdraht für die kanülierte und spitzenperforierte iliosakrale Schraube (Vollgewinde mit Unterlegscheibe) durch den entsprechenden iliosakralen Korridor platziert. Bei deutlich reduzierter Darstellbarkeit anatomischer Landmarken durch osteopene bzw. osteoporotische Knochenqualität empfiehlt sich die 3-D-navigierte Platzierung des Führungsdrahts. Alternativ kann die Lage des Führungsdrahtes vor dem Einbringen der Schrauben mittels 3-D-Scan überprüft und ggf. korrigiert werden. Nach Längenbestimmung und trikortikalem Überbohren des Drahtes wird die ausgemessene Schraube eingedreht (z. B. Fa. Marquardt Medizintechnik, Spaichingen, Deutschland, A Abb. 13). Sollte bei diesen Schritten (Bohren/Eindrehen) ein reduzierter Bohr- bzw. Eindrehwiderstand festgestellt werden, ist dies ein Hinweis auf eine reduzierte Knochenqualität und die Zementaugmentation der Schraubenspitze kann angeschlossen werden. Dazu wird mit einem Spezialinstrumentarium (z. B. Fa. Marquardt Medizintechnik, Spaichingen, Deutschland, > Abb. 14; alternativ mit einer Vertebroplastiekanüle, z. B. Fa. DePuy Synthes, Zuchwil, Schweiz, • Abb. 15) zunächst eine Kontrastmittelprobe durchgeführt (wasserlösliches Kontrastmittel unverdünnt oder 1:1 gemischt mit physiologischer NaCl-Lösung; z. B. Ultravist ${ }^{\circledR}$, Bayer Pharma AG, Berlin). Dabei sollte das Kontrastmittel ein regelrechtes Abfluten über den sakralen Venenplexus demonstrieren ( $\triangleright$ Abb. 16). Kommt es zu einer Vertei-
FFP1

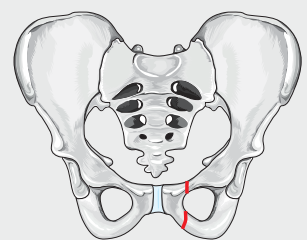

1a

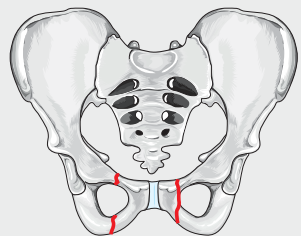

$1 b$

$2 b$

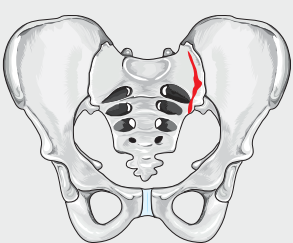

$2 a$

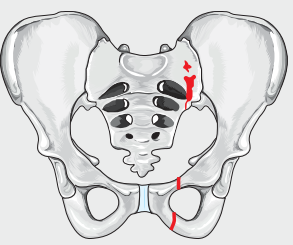

$2 b$

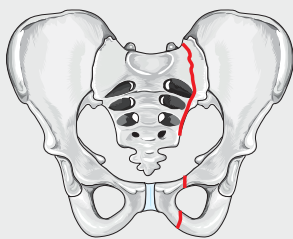

FFP3

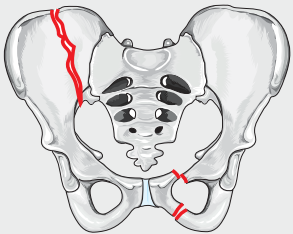

3a

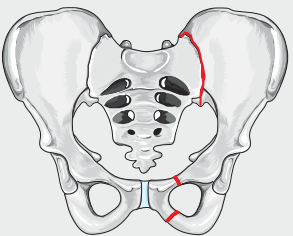

$3 b$

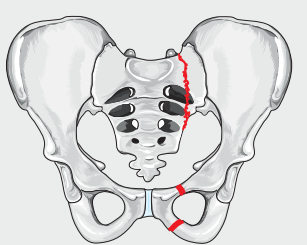

FFP4

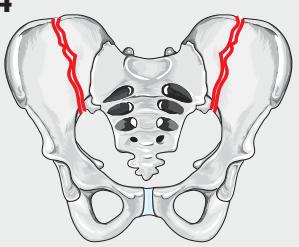

$4 a$

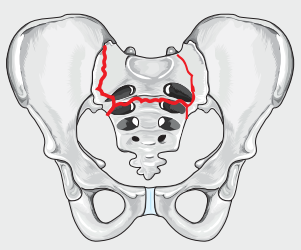

4b

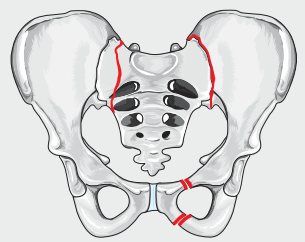

4b

- Abb. 12 Klassifikation der osteoporotischen Beckenringfrakturen (fragility fractures of the pelvis) nach Rommens [8] mit entsprechenden Untergruppen (http://dx.doi.org/10.1016/j.injury.2013.06.023, mit freundlicher Genehmigung des Elsevier Verlags). Aufsteigende Frakturinstabilität von Gruppe FFP 1 zu FFP 4. Charakteristika und Therapieoptionen siehe $>$ Tab. 1 bzw. Text. 
- Tab. 1 Tabellarische Darstellung der Klassifikation nach Rommens et al. für osteoporotische Beckenringfrakturen (Fragility Fractures of the Pelvis = FFP) mit Therapieoptionen [8].

\begin{tabular}{|c|c|c|}
\hline Einteilung & Charakteristika & Therapieoptionen \\
\hline Typ FFP 1 & $\begin{array}{l}\text { - isolierte unilaterale oder bilaterale Fraktur des vorderen Beckenrings } \\
\text { " stabil } \\
\text { - selten }\end{array}$ & - konservative Therapie \\
\hline Typ FFP 2 & $\begin{array}{l}\text { - einseitige nicht dislozierte Sakrumfraktur (transalar) mit/ohne ven- } \\
\text { trale Frakturkomponente } \\
\text { - moderat instabil } \\
\text { - meist akutes Trauma } \\
\text { - gehäuft bei beckennahen Prothesen/Implantaten }\end{array}$ & $\begin{array}{l}\text { - konservativer Therapieversuch } \\
\text { legitim } \\
\text { - operative minimalinvasive } \\
\text { Therapie (bei Versagen der } \\
\text { konservativen Therapie) }\end{array}$ \\
\hline Typ FFP 3 & $\begin{array}{l}\text { - dorsoventrale Fraktur (dorsal: transiliakal, transiliosakral, transsakral) } \\
\text { - hoch instabil } \\
\text { - Schmerzanamnese seit 4-6 Wochen } \\
\text { - Frakturdislokation/Kallusformation }\end{array}$ & $\begin{array}{l}\text { - meist offenes chirurgisches } \\
\text { Vorgehen erforderlich } \\
\text { - Kombination mit perkutanen } \\
\text { Verfahren möglich }\end{array}$ \\
\hline Typ FFP 4 & $\begin{array}{l}\text { - höchstgradig instabil } \\
\text { - bilaterale dorsale Fraktur (komplette spinopelvine Dissoziation) } \\
\text { - komplexe Frakturmorphologien (dorsal: transiliakal, transalar, sakral } \\
\text { U-förmig/H-förmig, ventrale Frakturkomponente) }\end{array}$ & $\begin{array}{l}\text { spinopelvine Osteosynthese } \\
\text { erforderlich }\end{array}$ \\
\hline
\end{tabular}

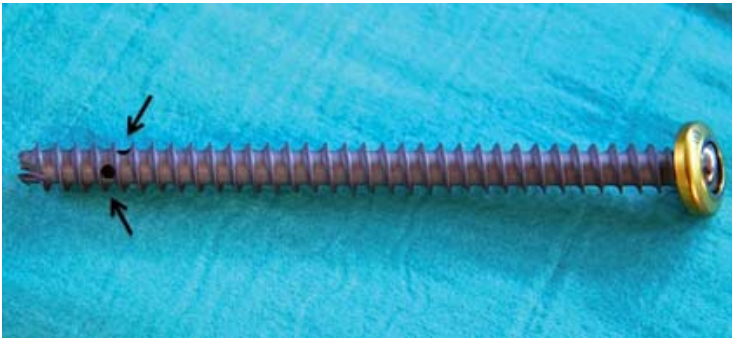

- Abb. 13 Kanülierte Schraube ( $\varnothing 7,3$ mm mit fest montierter Unterlegscheibe, Fa. Marquardt Medizintechnik, Spaichingen, Deutschland) mit Spitzenperforationen (Pfeilmarkierungen) für den Zementaustritt beim Augmentieren.

lung Richtung Spinalkanal, Neuroformamina oder intrapelvin, sollte keine Zementaugmentation durchgeführt werden, um eine kritische Zementfehlverteilung in Richtung wichtiger anatomischer Strukturen zu verhindern.

Die Schraubenaugmentation erfolgt anschließend durch Injektion von 2-3 ml Knochenzement (z. B. TraumacemV+ ${ }^{\circledR}$; Fa. DePuy Synthes, Solothurn, Schweiz) unter regelmäßigen Röntgenkontrollen ( $\bullet$ Abb. 14, 15, 17 und 18).

Bei zusätzlicher knöcherner Beteiligung des vorderen Beckenrings kann nach Wundverschluss ein supraazetabulärer Fixateur externe angelegt werden.

Über die postoperative Mobilisation sollte individuell entschieden werden. Kann vom Patienten eine Teilbelastung eingehalten werden, so ist diese einer Vollbelastung zum Schutz der Osteosynthese vorzuziehen (Teilbelastung mit 20 kg für 6 Wochen). In den meisten Fällen ist dies jedoch nicht möglich, sodass eine schmerzadaptierte Vollbelas-

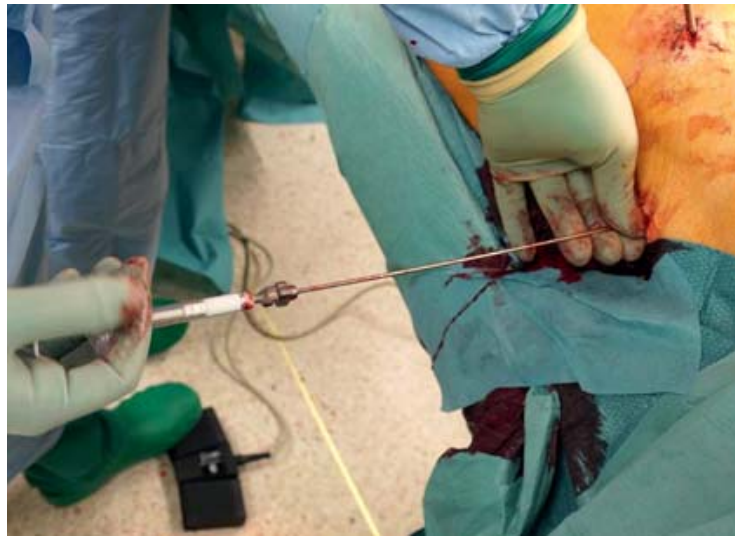

- Abb.14 Augmentationsinstrumentarium der Fa. Marquardt in der klinischen Anwendung. Die Länge der Kanüle erlaubt die Zementaugmentation auch bei größerem Weichteilmantel.

tung einer Rollstuhlmobilisierung vorzuziehen ist. Die Entfernung eines eingebrachten Fixateur externe kann 6-8 Wochen postoperativ nach einem Vollmobilisationsversuch erfolgen ( $\triangleright$ Abb. 19). Die eingebrachte augmentierte SI-Schraube verbleibt in der Regel in situ.

\section{Komplikationen}

Beim älteren Patienten kommen bevorzugt perkutane operative Verfahren in der Versorgung von Beckenringfrakturen zur Anwendung. Dadurch sollen Komplikationen aufgrund ausgedehnter operativer Zugänge vermieden und eine zügige Mobilisation gewährleistet werden.

Zu den intraoperativen Komplikationen der zementaugmentierten iliosakralen Verschraubung zählen Verletzun- 


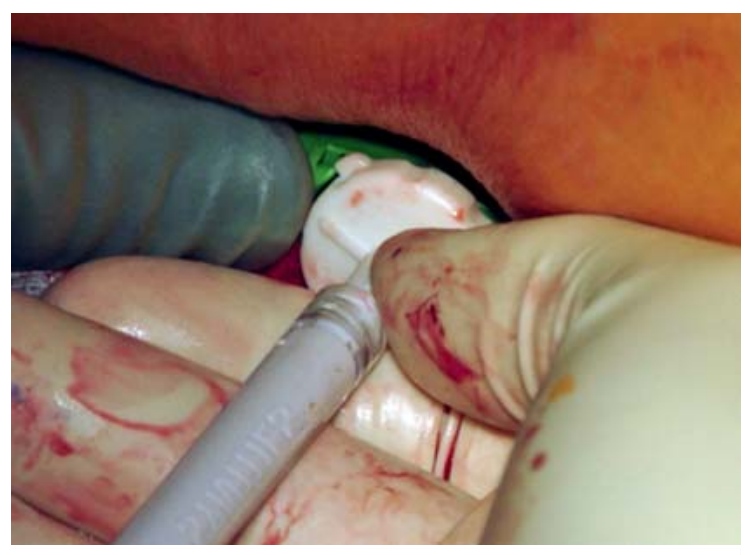

- Abb. 15 Klinische Darstellung des Augmentationsvorgangs über eine eingebrachte Vertebroplastiekanüle.

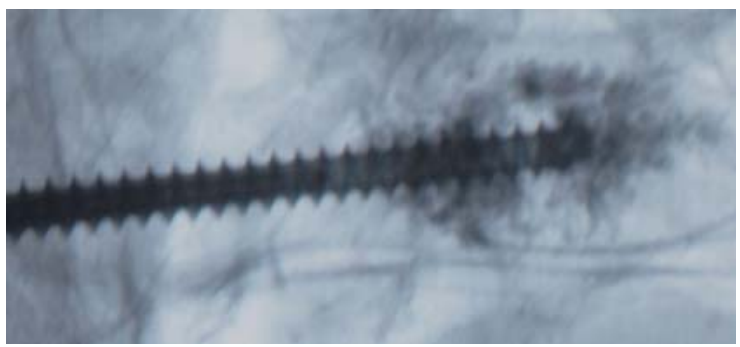

- Abb.17 Zementaugmentation der eingebrachten iliosakralen Schraube unter Bildwandlerkontrolle.

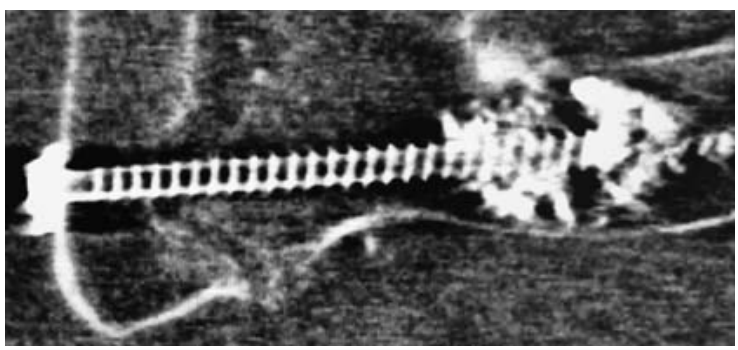

- Abb. 18 Kontrollbild (3-D-Scan mittels 3-D-Bildwandler) der eingebrachten Schraube inklusive Augmentationszement an Schraubenspitze.

gen von Glutealarterien, intrapelviner Gefäße, Spinalnerven ( $L$ V und S I), intraabdomineller Hohlorgane sowie der Cauda equina durch Fehlplatzierung des Zieldrahtes bzw. durch Schraubenfehllage. Hier hilft die 3-D-Navigation, um diese Komplikationen zu vermeiden. Bei reduzierter Knochenqualität erhöht sich das Risiko, die Schraube zu überdrehen oder mit der Unterlegscheibe in die Iliumkortikalis einzubrechen, dies resultiert in einem erheblichen Stabilitätsverlust. Zur Vermeidung dieser Komplikation

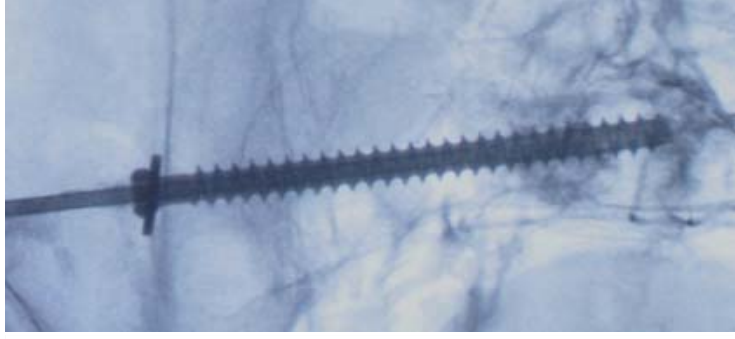

- Abb. 16 Kontrastmittelprobe zeigt reguläres Abfluten über perisakrale Venen (ventral und dorsal) ohne atypische Verteilung im Knochen Richtung Fraktur bzw. anatomischer Strukturen.

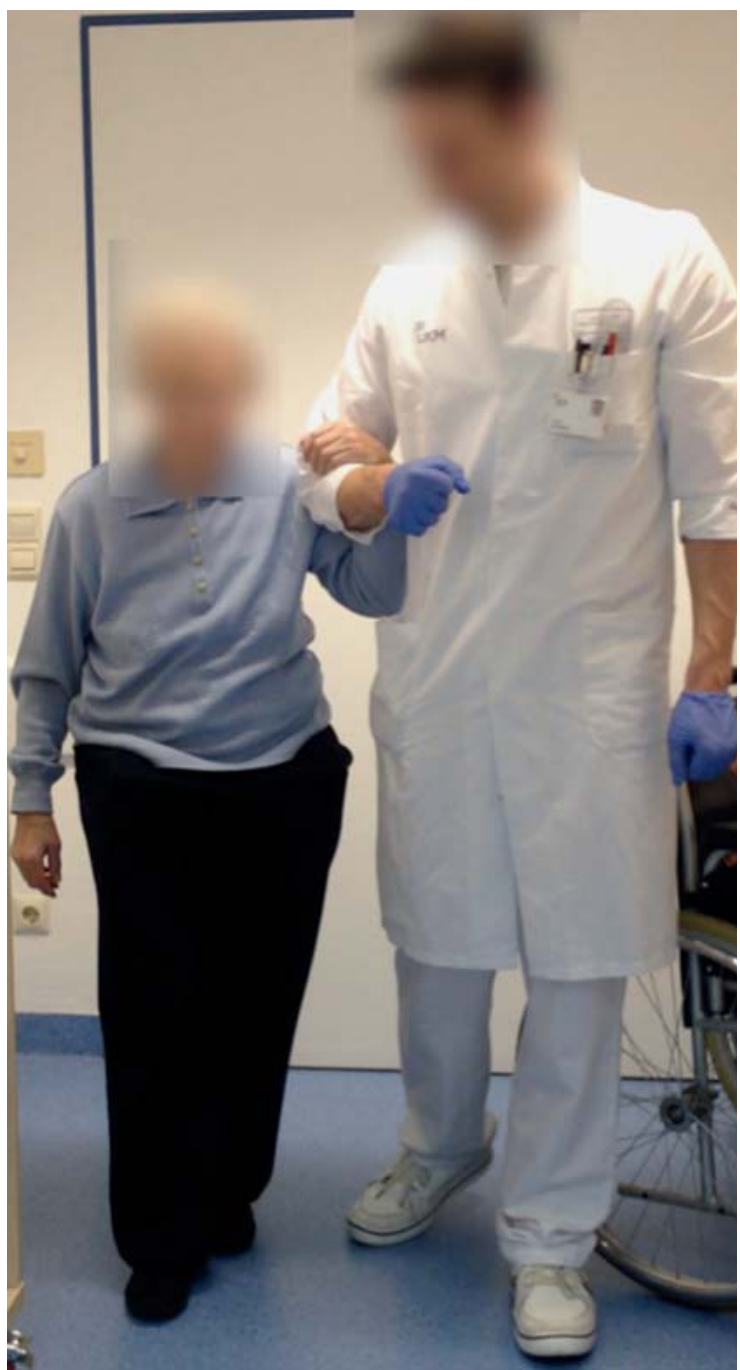

- Abb. 19 Vollmobilisationsversuch der o.g. 91-jährigen Patientin 6 Wochen postoperativ. Nach Lösen der Querstange des Fixateur externe erfolgt die in diesem Falle schmerzfreie Mobilisierung. 
nutzen wir Vollgewindeschrauben; eine Kompression der Frakturzone ist nicht erforderlich.

Während der Augmentation kann Knochenzement in das Gefäßsystem eindringen und im Extrem zu einer Zementembolie führen. Dies entspricht einer potenziell schweren Komplikation und bedarf eines sofortigen interdisziplinären Komplikationsmanagements. Deshalb ist vor der Injektion von Knochenzement das regelrechte Abfluten des Kontrastmittels zu überprüfen.

Postoperative Komplikationen umfassen Thrombosen, Embolien, Pneumonien und Dekubiti; weiterhin ist es wichtig, ein mit erhöhter Mortalität verbundenes postoperatives Delir durch einen multimodalen Therapieansatz zu verhindern [12].

Auch ein Implantatversagen mit einem Auslockern der Schraube trotz Zementaugmentation ist denkbar.

Unter Berücksichtigung des beschriebenen Vorgehens ist die minimalinvasive augmentierte SI-Verschraubung zur Therapie einer Fraktur des hinteren Beckenrings beim älteren Patienten ein sicheres, aber technisch aufwendiges Verfahren.

\section{Interessenkonflikt}

Prof. Raschke und PD Dr. Fuchs waren an der Entwicklung der kanülierten und fenestrierten Schraube der Firma Marquardt beteiligt.

Über die Autoren
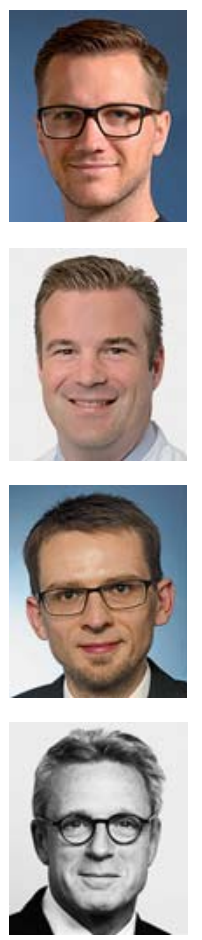

Niklas Grüneweller

Dr. med., wissenschaftlicher Mitarbeiter, Klinik für Unfall-, Hand- und Wiederherstellungschirurgie, Universitätsklinikum Münster

\section{Thomas Fuchs}

PD Dr. med., Klinikdirektor Unfall- und Wiederherstellungschirurgie, Vivantes Klinikum im Friedrichshain, Berlin

\section{Dirk Wähnert}

PD Dr. med., wissenschaftlicher Mitarbeiter, Klinik für Unfall-, Hand- und Wiederherstellungschirurgie, Universitätsklinikum Münster

\section{Michael J. Raschke}

Univ.-Prof. Dr. med. Klinikdirektor, Klinik für Unfall-, Hand- und Wiederherstellungschirurgie, Universitätsklinikum Münster

\section{Korrespondenzadresse}

\section{Prof. Dr. med. Michael Raschke}

Klinikdirektor

Klinik für Unfall-, Hand- und Wiederherstellungschirurgie

Universitätsklinikum Münster

Albert-Schweitzer-Campus 1

48149 Münster

Michael.Raschke@ukmuenster.de

Literatur

[1] Culemann U, Scola A, Tosounidis G et al. [Concept for treatment of pelvic ring injuries in elderly patients. A challenge]. Unfallchirurg 2010; 113: 258-271

[2] Fuchs $T$, Rottbeck $U$, Hofbauer $V$ et al. [Pelvic ring fractures in the elderly. Underestimated osteoporotic fracture]. Unfallchirurg 2011; 114: 663-870

[3] Gänsslen A, Hüfner T, Krettek C. Percutaneous iliosacral screw fixation of unstable pelvic injuries by conventional fluoroscopy. Oper Orthop Traumatol 2006; 18: 225-244

[4] Hadji P, Klein S, Häussler B et al. The bone evaluation study (BEST): patient care and persistence to treatment of osteoporosis in Germany. Int J Clin Pharmacol Ther 2013; 51: 868-872

[5] Pohlemann T, Stengel D, Tosounidis G et al. Survival trends and predictors of mortality in severe pelvic trauma: estimates from the German Pelvic Trauma Registry Initiative. Injury 2011; 42: 997-1002

[6] Rommens PM, Wagner D, Hofmann A. [Osteoporotic fractures of the pelvic ring]. Z Orthop Unfall 2012; 150: e107-e118

[7] Rommens PM, Wagner D, Hofmann A. Surgical management of osteoporotic pelvic fractures: a new challenge. Eur J Trauma Emerg Surg 2012; 38: 499-509

[8] Rommens PM, Hofmann A. Comprehensive classification of fragility fractures of the pelvic ring: recommendations for surgical treatment. Injury 2013; 44: 1733-1744

[9] Scheyerer M], Osterhoff G, Wehrle S et al. Detection of posterior pelvic injuries in fractures of the pubic rami. Injury 2012; 43: $1326-1329$

[10] Stubbs B, Brefka S, Denkinger MD. What works to prevent falls in community-dwelling older adults? Umbrella review of meta-analyses of randomized controlled trials. Phys Ther 2015; 95: 1095-1110

[11] Studer P, Suhm N, Zappe B et al. Pubic rami fractures in the elderly - a neglected injury? Swiss Med Wkly 2013; 143: w13859

[12] Wähnert D, Roos A, Glasbrenner J et al. [Traumatology in the elderly: Multimodal prevention of delirium and use of augmentation techniques]. Chirurg 2017; 88: 95-104

Bibliografie

DOI http://dx.doi.org/10.1055/s-0043-103763

OP-JOURNAL 2017; 33: 18-26 @ Georg Thieme Verlag KG Stuttgart · New York ISSN 0178-1715 